\title{
Quelques remarques sur le traitement du placenta pravia.
}

\author{
Par M. ELIS ESSEN-MÖLLER, Lund.
}

La discussion relative au traitement du placenta pravia n'est pas encore terminée, ce qui n'est pas, du reste, difficile ¿i comprendre. Les procédés sont nombreux et les opinions des acconcheurs sur leur valeur relative varient. Aussi, les cas varient et exigent telle ou telle autre méthode, dont les indications ne sont pas toujours faciles à préciser. Ces derniers temps, on a vu naître un mouvement en faveur de la section césarienne, abdominale ou vaginale. Si l'on considère en outre que l'insertion vicieuse constitue une complication des plus graves, pour les mères comme pour les enfants, et qu'elle se présente au praticien comme au clinicien, il est facile de comprendre que l'interêt an sujet du traitement du previa soit toujors vif.

A Ia clinique des femmes de la faculté de médecine de Lund, j’ai observé 132 cas de placenta provia. Pour les in. dications du traitement, je les ai posées moi-même, ainsi que j’ai traité personellement la plupart des cas. Done, ma statistique ne manque pas d'unité. Cependant, pendant les vingt amnées que comportent mes observations, mon opinion sur le traitement du provia a sensiblement changé depuis l'année: 1916 ; cest pourquoi je désire soumettre maintenant mes cas aux accoucheurs étrangers.

D'abord quelques remarques sur les règles qui sont ì la base de ma statisticue. J'ai compté tous les cas, même ceux

1 2013ir. Artu Gypecologina Iot. I. Fuse. 1. 
où la mère fut amenée mourante, où une traîtement ne pût la sauver. J'ai aussi compté comme morts, sans déduction, tous les cas où l'enfant était mort avant l'accouchement, pendant l'accouchement ou après la naissance, tant que la mère était encore à la clinique. On me dira peut-être qu'une telle statistique donne une mortalité trop élevée, et c'est peut-être vrai. Mais je me méfie des statistiques réduites", qui le sont souvent un peu plus qu'il n'est compatible avec l'objectivité. Je pense, avec M. DöDerLein, qu'il raut mieux compter la mortalité en bloc, même si l'on court le risque de compter un cas où la mort n'est pas due au traitement, plutôt que de commettre la faute plus grande d'effacer la vérité par une réduction subjective.

C'est pourquoi j'ai compté aussi les enfants prématurés morts. J'ai pensé que, dans des statistiques assez considérables, ces cas se rencontrent environ dans le nême nombre relatif. Pour rendre possible, cependant, la comparaison de mes résultats à ceux ou les enfants prématurés sont déduits, j'ai donné aussi les chiffres des enfants de plus de 2,000 grammes de poids.

Le résultat de mes 132 cas donne 5 cas de morts, soit $3,7 p$. C., pour les mères, et une mortalité fétale de 63 cas, soit 45,6 p. C. (6 fois grossesse gémallaire).

Les 5 cas de mort de la mère sont dus à 1 : tamponnement à la maison, infection; 2: anémie et infection après version et délivrance artéficielle du placenta; 3: infection avec pbysomètre ì l'arrivée, mort en une demi-heure; 4: anémie après ponction des membranes et extraction; 5: embolie pulmonaire après section césarienne abdominale.

Nous allons examiner maintenant les différents procédés employés.

L'accouchement a été spontané dans 22 cas avec toutes les mères virantes et 8 enfants morts $=36,3 \mathrm{p}$. C. Si l'on ne considère que les enfants de 2,000 grammes et plus, la mortalité foetale a été de 3 eas sur $15=20$ p. C. Il va sans dire que tous les cas spontanés ont été des cas légers.

La ponction des membranes seule a été pratiquée dans 33 cas. Le résultat pour les mères a été le meilleur possible, 
O. p. C. de mortalité. Pour les enfants, la mortalité a été de 28,4 C. (10 sur 34, une fois des jumeaux). Avec déduction des prématurés de moins de 2,000 grammes, la mortalité a été de 23,3 p. C.

Dans deux autres cas, la perforation des membranes a été suivie d'une application du forceps (un enfant vivant, un mort).

Pendant ces dernières années, la ponction des membranes a été toujours suivie d'une injection de pituitrine, ce qui, d'après mon opinion, a en un effet très avantageux sur le travail, qui, comme on sait, traîne souvent en longueur.

Nous considérons alors les résultats de la version diapris Braxton-Hicks. Sur 51 mères qui ont subi cette intervention, 2 ont succombé, les causes de la mort ont été lues plus haut. Tontes les deux étaient perdues déjà à l'arrivée à la clinique; autrement je crois que la version elle-même ne doit pas entrainer de mort chez une femme non infectée et non ané. miée, si elle est pratiquée par une moin expérimentée et aseptique. Des 53 enfants (deux fois jumeaux) 37 ont succombé, soit 69,8 p. C. Après déduction des prématurès, il y avait 27 morts sur $4 t=61,9$ p. C.

Je tiens à dire ici qu'à la clinique de Lund la version a été le procédé de choix et que, par principe, j'ai insisté toujours sur ce que l'accouchement après lintervention füt spontané, sans extraction avant que l'orifice ne fut complètement dilaté. Je reviendrai plus tard sur ce point. A ce point de vue, il est intéressant de constater que, sur 42 enfants dont l'accouchement a été spontané après la version, 31 sont morts, soit 73,8 p. C., tandis que sur les 11 quion pouvait extraire immédiatement après la version 6 seulement étaient morts, soit 54,5 p. C. Avec déduction des prématurés, les chiffres correspondants sont de 68,5 et $16,6 \mathrm{C}$.

Le tamponnement vaginal n'a été pratiqué que 8 fois dont deux fois seulement à la clinique, les autres à la maison avant l'arrivée de la malade à la clinique. Je passe là-dessus, parceque j'estime le tamponnement vaginal dans les cas de placenta prævia comme un procédé dangereux et condamnable. 
La saisie d'un pied dans la présentation du siège a été pratiquée quatre fois, dont une fois avec un foetus mort.

Viennent ensuite les deux interventions chirurgicales, l'opération césarienne abdominale et vaginale, dont la place dans le traitement du previa est si vivement discutée et contestée. J'ai fait sept césariennes abdominales pour placenta provia. Une des mères a succombé d'une embolie d'air, peu de temps après l'opération. Malheureusement, je n'ai pas obtenu la permission d'une nécroscopie, mais tout le syndrome ne se laisse pas expliquer d'une autre manière. Une telle complication étant connue dans le placenta prævia après une version podalique, il est difficile de juger jusqu'à quel degré l'opération césarienne comporte un risque plus grand, à ce point de vue. Mais j'insiste sur ce qu'un tel accident doit être un memento pour ceux qui sont inclinés à étendre les indications de la césarienne abdominale pour pravia, d'après l'exemple de MM. Krönig et Seluheim. - Parmi les enfants, deux ont succombé. L'un était mort avant l'opération qui fut prati¿uée pour sauver la mère, presque sans pouls, le col fermé. L'autre était prématuré et n'a vécu qu'une heure.

Il y a eu 12 opérations césariennes vaginales. Toutes les mères sont sorties guéries et il n'y a eu qu'un enfant de mort. Dans sept cas, l'intervention a été pratiquée sans intervention préalable. Dans les trois autres je l'ai fait secondairement (après version, perforation des membranes et version avec procidence du cordon), quand les battements du coeur ont démontré que le foetus commençait à souffrir, done exclusivement dans l'interêt de l'enfant. Les trois enfants ont été sauvés et je tiens à dire que celà aurait été impossible sans la section rapide du ségment inférieur et l'extraction immédiate.

J'ai recherché dans la littérature les résultats de la césarienne vaginale pour prævia, obtenus dans les cliniques où l'on a pratiquée cette opération plus souvent que dans des cas isolés. Voici ce que j’ai trouvé. 


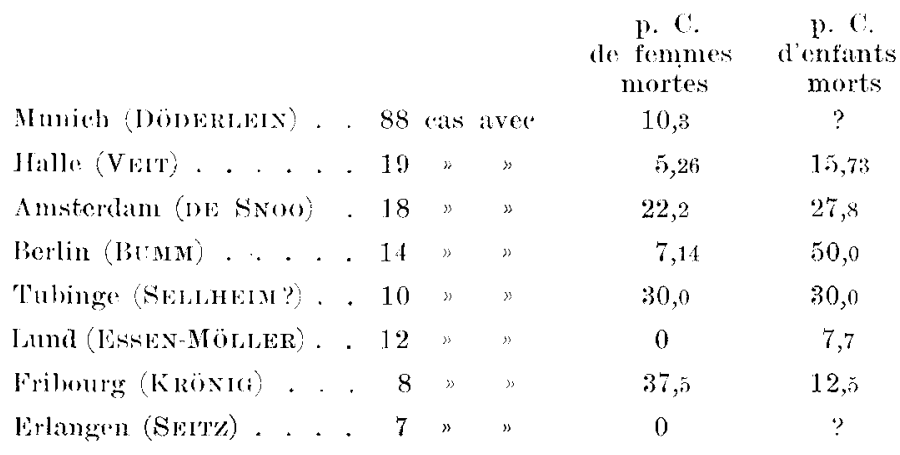

Ayant exposé maintenant mes résultats, il me reste à faire quelques remarques sur le traitement, tel qu'il mé paraît devoir être pratiqué. La première se rapporte aux mères.

Il est généralement admis que les risques du placenta pravia, wu point de vue de la mère, sont contenus dans ces deux mots: l'hémorragie et l'infection. Je ne sauraris dire laquelle des deux est la plus grave; seulement on ne peut pas nier que la résistance contre l'infection va en diminuant à mesure que la mère a perdu du sang. A ce point de vue, le but principal doit être de târir l'hémorragie. Or, celà doit être effectué, avant tout, de telle manière que la femme soit remise ¿ une rlinique spéciale ou à une maternité le plus tôt possible après la première hémorragie. Il ne doit pas suffir, à l'avenir, de laisser venir une hémorragie après l'autre, tout en esperant que la femme se rétablira de son anémie après chaque perte. Les hémorragies surviennent de nouveau à des intervalles plus rapprochés et la femme succombera peut-être un jour d'une perte rélativement peu abondante.

D'autre part, si la femme est admise après la première hémorragie, il appartiendra au clinicien de juger, si un traitement immédiat est nécessaire on si l'on peut attendre. Mais, en tout cas, l'observation permanente de la malade est de rigueur. On ne peut nier que les résultats qui seront obtenus de cette manière ne soient meilleurs que ceux obtenus auparavant.

Entre autres, on aura l'avantage de pouvoir abolir com- 
plètement le tamponmement vaginal. Il est rare que la première hémorragie soit abondante. Donc, une fois qu'elle aura cessé, le medecin ou la sage-femme remettra la femme à la clinique, sans tamponmement. Si, comme le dit M. BAR, l'infection est la cause principale de la mort dans ces cas-là, on aura done la possibilité de la diminuer, de cette façon, d'une manière efficace.

La seconde remarque se rapporte aux resultats concernant les enfants. On voit, d'après ce qui précède, que les résultats de la version sont excellents pour la mère, mais misérables pour les enfants. Si l'on me demande pourquoi je n'ai pas recouru à la métreuryse, j'avouerai que je n'admire pas cette méthode. Il est difficile de placer le ballon dans la cavité ovulaire; souvent on échoue et l'introduit entre les membranes ct l'utérus, ce qui augmente le décollement de placenta. De phus, il faut, après la dilatation complète, recourir à la version, ce qui sionifie une nouvelle intervention. Enfin, l'instrument est fragile. Je ne trouve pas non plus que la métreuryse domne de meilleurs résultats pour la mère, et je crois que la supériorité des résultats concernant les enfants n'est jas si considérable qu'elle ne soit pas à atteindre par une autre voie.

En cherchant done un procédé qui, tout en donnant des résultats favorables pour la mère, pourrait améliorer le sort des enfants, je me suis arrêté devant le fait que la mortalité des enfants nés spontanément après la version a été de 73,8 1. C. alors que les résultats qui ont été obtenus pour les en fants extraits immédiatement après la version ont été bien meilleurs, à savoir de $54,5 \%$. Sans les prématurés, les chiffres ont été de 68,5 et de $16,6 \mathrm{p}$. C.

Il faudrait done recourir à des méthodes qui, tout en sauvegardant la mère, rendent possible l'extraction immédiate de l'enfant. C'est dans ce but que je me suis servi des méthodes chirurgicales.

Fin ce qui concerne l'opération césarienne abdominale, quoique je l'aie pratiqué sept fois pour previa, je ne crois pas que cette opération ait jamais une grande application dans 
le traitement de l'insertion viciense. Comme le dit $\mathrm{M}$. BrINDFAU ${ }^{1}$, lopération est "logique puisqu'elle permet de vider rapidement l'utérus suns intéresser le segment inférieur», mais comme lui je me borne à la pratiquer dans les cas à col fermé non infectés, ò l'hémorragie est abondante. Mais dans de tels cas je crois juste d'intervenir dans l'intèrêt de la mère autant quo dans celui de l'enfant. Ainsi, dans un de mes cas, j’ai opéré bien que l'enfant fût mort avant l'opération. L'hémorragie par le col fermé était foudroyante; la mère fut guérie.

Mais si l'on est d'accord sur l'indication rare et exceptionelle de la césarienne abdominale, il faut recourir à d'autres méthodes pour faciliter la naissance immédiate du foetus souffrant. C'est dans ce but que j'ai pratiqué dix fois la césarienne vaginale pour placenta previa.

Comme je l'ai mentionné déjà, l'opération fut pratiquée, dans sept cas, avant toute autre intervention et fut suivie de la version et de l'extraction. Dans les trois derniers cas, j'ai commencé par la version, et quand le foetus a commencé à souffrir jai sectionné le segment inférieur, toujours dans la paroi antérieure. On a done l'avantage que le membre abaissé et le sioge forment un tampon très efficace de la plaie utérine, et qu'on n'a pas besoin d'allonger l'incision plus haut que ce n'est nécessaire pour permettre tout juste l'issue de l'enfant. Tmmédiatement après la naissance de l'enfant on peut poser les sutures. J'ai été étonné de l'hémorragie peu considérable observée dans mes cas.

Je ne doute pas qu'il y ait des collègues qui ne trouvent inconséquent qu'un accoucheur qui a, de crainte d'une rupture cervicale, toujours exigé l'accouchement spontane après la version, pratique maintenant à propos délibéré une incision justement dans cette partie de l'utérus où est situé le placenta et d'où l'on pent attendre une hémorragie formidable. Aussi, les risques théoriques sont évidents et personne ne peut les mépriser. Je sais aussi très bien que d'éminents opéra-

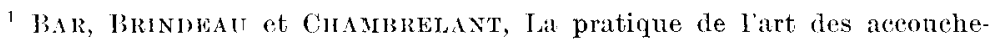
ments. 3ue Ed. P'aris 1914. Tome second, p. 480. 
teurs ont échoué et quaprès des issues fatales ils déconseillent lopération. Aussi, je l'avoue franchement, je ne sais pas moi-même si l'opération est justifiée; seulement, j’ai essayé l'améliorer les résultats pour les enfants, et je suis heureux que celà m'ait réussi sans dommage pour les mères.

Au point de vue anatomique, il paraît vraisemblable que l'hémorragie due à l'incision doive être plus abondante à mesure que cette incision tombe sur le placenta, c'est-i-dire que l'incision de la paroi utérine antérieure doit amener une hémorragie plus profuse si le placenta $y$ est situé que s'il est situé sur la paroi postérieure. Aussi, M. Brindeau, dans le traité cité, ne la conseille que dans les cas limités aux insertions postérieures. Malheureusement, il est souvent très difficile ou même impossible de poser le diagnostic exact de l'insertion placentaire; souvent on rencontre partout le tissu placentaire. Comme je ne me sens pas incliné à suivre l'exemple de M. Krönia qui voudrait faire l'incision de la paroi postérieure de l'utérus, je crois inévitable de sectionner la paroi antérieure dans tous les cas où l'on aura recours à cette opération. Il me semble alors prudent de faire dabord la version et après elle la césarienne vaginale ou une incision profonde de DüHrssen, d'après les circonstances.

Je mabstiendrai des raisonnements théoriques, et je vous prie de considérer simplement les résultats: toutes les femmes vivantes, un unfant mort-né. Mais j'insiste en outre sur un autre résultat. Axant ma première opération céserionne vaginale pour provia, la mortalité des enfants a été de 58, p. C.; apris cette date, où les enfants ont été extraits immédiatement, grice amx intercentions chirurgicales, elle s'est abaissé à 20,4 $p$. (', sans que les résultats pour les mères en aient été modifies. Done je crois qu'il me sera permis de poursuivre cette voie, tout en avouant que l'avenir pourrait causer des déceptions.

Ayant plaidé pour l'admission immédiate de la malade dans une clinique dès la première hémorragie, j’ai accentué la difference entre l'art des accouchements telle qu'on le pratique daus la famille de la malade et l'obstétrique moderne des maternités et des hôpitaux. On m'opposera péutêtre qu'un 
accoucheur habile pourra maitriser les cas autant chez la malate ellemème qu'à l'hôpital. Je l'admets volontiers pour les cas légers. Pour les graves complications, je crois cependant que les progrès modernes, réalisés par exemple pour les cas de bassins rétrécis, d'éclampsie, de rupture utérine et maints autres, n'auraient été possibles que dans des cliniques spéciales. Celì est généralement admis, non seulement par les accoucheurs, mais aussi par les sages-femmes elles-mêmes, dont nous avons vu sonvent le soulagement lorsqu'elles ont pu envoyer une malade à une maternité, ce qui les débarasse d'une lourde responsabilité. Ce que je voudrais soutenir, c'est que les cas de placenta provia rentrent dans la catégorie des cas, où l'hôpital donne de meilleurs résultats que le traitement i la maison. Plus les cas seront traités dans un hôpital et avant que la malade n'ait perdu beaucoup de sang, plus les résultats iront en s'méliorant, tout pour la mère que pour l'enfant. 